\title{
Epidemiology and health outcomes of sarcoidosis in a universal healthcare population: a cohort study
}

\author{
Lee M. Fidler ${ }^{1,2,3}$, Meyer Balter ${ }^{4}$, Jolene H. Fisher ${ }^{1}$, Teresa To ${ }^{5,6}$, \\ Matthew B. Stanbrook ${ }^{1}$ and Andrea Gershon $\mathbb{1}^{2,3,5}$ \\ Affiliations: ${ }^{1}$ Division of Respirology, Dept of Medicine, University Health Network, Toronto, ON, Canada. \\ ${ }^{2}$ Division of Respirology, Dept of Medicine, University of Toronto, Toronto, ON, Canada. ${ }^{3}$ Division of \\ Respirology, Dept of Medicine, Sunnybrook Health Sciences Centre, Toronto, ON, Canada. ${ }^{4}$ Division of \\ Respirology, Dept of Medicine, Mount Sinai Hospital, Toronto, ON, Canada. ${ }^{5}$ Institute for Clinical Evaluative \\ Sciences, Toronto, ON, Canada. ${ }^{6}$ Child Health Evaluative Sciences, The Hospital for Sick Children, Toronto, \\ ON, Canada.
}

Correspondence: Lee M. Fidler, Division of Respirology, Dept of Medicine, Room A459, Sunnybrook Health Sciences Centre, 2075 Bayview Avenue, Toronto, ON, M4N 3M5, Canada. E-mail: lee.fidler(autoronto.ca

@ERSpublications

The prevalence of sarcoidosis in Canada is increasing, with an estimated rate of 143 per 100000 as of 2015. The burden of sarcoidosis is changing, with increasing incidence among males in recent years and decreasing disease rates in females. http://bit.ly/2K4WxP5

Cite this article as: Fidler LM, Balter M, Fisher JH, et al. Epidemiology and health outcomes of sarcoidosis in a universal healthcare population: a cohort study. Eur Respir J 2019; 54: 1900444 [https://doi.org/ 10.1183/13993003.00444-2019].

ABSTRACT Sarcoidosis-related mortality appears to be rising in North America, with increasing rates in females and the elderly. We aimed to estimate trends in sarcoidosis incidence, prevalence and mortality in Ontario, Canada.

We performed a cohort study using health administrative data from Ontario between 1996 and 2015. International Classification of Diseases and Ontario Health Insurance Plan codes were used for case detection. Three disease definitions were created: 1) sarcoidosis, two or more physician claims within 2 years; 2) chronic sarcoidosis, five or more physician claims within 3 years; and 3) sarcoidosis with histology, two or more physician claims with a tissue biopsy performed between claims.

Overall, 18550, 9199 and 3819 individuals with sarcoidosis, chronic sarcoidosis and sarcoidosis with histology, respectively, were identified. The prevalence of sarcoidosis was 143 per 100000 in 2015, increasing by $116 \%(\mathrm{p}<0.0001)$ from 1996 . The increase in age-adjusted prevalence was higher in males than females $(136 \%$ versus $99 \%$; $\mathrm{p}<0.0001)$. The incidence of sarcoidosis declined from 7.9 to 6.8 per 100000 between 1996 and 2014 (15\% decrease; $\mathrm{p}=0.0009)$. A 30.3\% decrease in incidence was seen among females $(\mathrm{p}<0.0001)$ compared with a $5.5 \%$ increase in males $(\mathrm{p}=0.47)$. Age- and sex-adjusted mortality rates of patients with sarcoidosis rose from $1.15 \%$ to $1.47 \%$ between 1996 and 2015 (28\% increase; $p=0.02$ ), with the overall trend being nonsignificant $(p=0.39)$. Mortality rates in patients with chronic sarcoidosis increased significantly over the study period ( $\mathrm{p}=0.0008)$.

The prevalence of sarcoidosis is rising in Ontario, with an apparent shifting trend in disease burden from females to males. Mortality is increasing in patients with chronic sarcoidosis. 


\section{Introduction}

Sarcoidosis is a multisystem granulomatous disease of unknown cause [1]. The epidemiology of sarcoidosis has differed across studies, likely as a result of varied environmental factors, genetic susceptibility and study design [1-3]. Recent national database studies focused on the epidemiology of sarcoidosis in the USA have been insightful, but restricted to individuals with health insurance; this may misrepresent the epidemiology of sarcoidosis in the general population, as sarcoidosis is reportedly more common in African-Americans, an ethnic group more likely to lack health insurance $[1,4,5]$. Research using complete population databases is needed for accurate disease estimates. Knowing the epidemiology and mortality trends of sarcoidosis is important to inform patients, healthcare providers and decision makers to allow adequate healthcare planning. Furthermore, little is known of the epidemiology of sarcoidosis in Canada.

There are over 14 million people living in Ontario with coverage under its universal healthcare system [6]. Using health administrative data, we aimed to estimate trends in prevalence, incidence and mortality of sarcoidosis in this large, complete Canadian population, a population reflective of many other areas of North America. Additional aims were to describe patient demographics and medication use of individuals with sarcoidosis $\geqslant 65$ years of age.

\section{Methods}

\section{Data sources}

We performed a population-based cohort study using health administrative data to estimate the incidence, prevalence and mortality of sarcoidosis in Ontario, Canada. Physician claim diagnoses, including both inpatient and outpatient claims by primary care physicians and specialists, were obtained from the Ontario Health Insurance Plan (OHIP) records. OHIP covers all medical necessary services in the province of Ontario. The Canadian Institute of Health Information Discharge Abstract Database (www.cihi.ca/en/ discharge-abstract-database-metadata) was utilised to obtain information regarding hospital, day-unit and emergency department discharges. Demographic data, including patient deaths and date of entering/ leaving the province, were obtained from the Ontario Registered Persons Database (www.ontario.ca/data/ registered-persons-database-rpdb). Medication prescription records for patients $\geqslant 65$ years of age were obtained from the Ontario Drug Benefit Program database (www.ontario.ca/data/ontario-drug-benefitodb-database). Data was linked on an individual level using encrypted health card numbers. Research ethics approval was obtained from the Institutional Review Board of Sunnybrook Health Sciences Centre (Toronto, ON, Canada).

\section{Study design and participants}

All adults (aged 18-105 years) living in Ontario between 1991 and 2015 were included. Those without a valid health card number, not present in the registered persons database, living outside of Ontario or living in Ontario for $<1$ year were excluded. Sarcoidosis was identified using International Classification of Diseases, Clinical Modification (ICD)-9/10 codes from outpatient and hospitalisation physician claims specific to sarcoidosis (ICD-9: 135 (sarcoidosis) or 3214 (sarcoid meningitis); ICD-10 sarcoidosis-specific codes: D86.0 (lung), D86.1 (lymph nodes), D86.2 (lung and lymph nodes), D86.3 (skin), D86.8 (other and combined sites), D86.9 (unspecified), G53.2 (cranial nerve palsies in sarcoidosis) or M63.3 (myositis in sarcoidosis)) and an OHIP diagnostic code (135 (sarcoidosis)). The switch from ICD-9 to ICD-10 coding took place in Ontario in 2002.

Three sarcoidosis case definitions were created: sarcoidosis, chronic sarcoidosis and sarcoidosis with histology. Sarcoidosis was defined as two or more physician claims or hospitalisations for sarcoidosis, at least 2 weeks apart but within 2 years. This definition is similar to previously published epidemiological studies $[4,7]$. A sarcoidosis disease definition requiring two or more sarcoidosis-coded claims has been previously reported to identify $95 \%$ of patients with sarcoidosis in a well-characterised clinical cohort [7]. Patients with an increased number of physician claims for sarcoidosis-specific codes were suspected to be more likely to have chronic sarcoidosis, given an ongoing need for medical evaluation. Chronic sarcoidosis was defined as five or more physician claims or hospitalisations for sarcoidosis, at least 2 weeks apart but within 3 years. Patients with sarcoidosis who underwent a tissue biopsy during their evaluation were felt to represent an important subgroup. Sarcoidosis with histology was defined as two or more physician claims or hospitalisations for sarcoidosis plus the performance of a tissue biopsy. If there were multiple physician claims on the same day, only one was counted. Physician claims had to be separated by at least 2 weeks and at least one of the sarcoidosis physician claims for sarcoidosis with histology cases had to occur after the tissue biopsy date. OHIP tissue biopsy codes included: endobronchial ultrasound and biopsy (G050), transbronchial biopsy (E638, Z333), mediastinoscopy (Z329), lung biopsy (Z338, M137), lymph node biopsies (M138, Z405, Z411, Z406), liver biopsy (Z551), and skin biopsy (Z116, Z113). As we did not confirm with certainty that patients meeting the developed disease definitions had the disease, the specific titles ascribed to the three case definitions (sarcoidosis, chronic sarcoidosis and sarcoidosis with histology) 
were applied for ease of description. The study investigators believed the case definitions to have face validity, given the ICD-9/10 codes are specific to sarcoidosis and repeated visits were required. The three sarcoidosis case definitions were also believed to have internal validity, allowing comparisons between disease subgroups. The first sarcoidosis physician claim date was labelled as the date of diagnosis. Given there is no cure for sarcoidosis, patients fulfilling any case definition remained part of our study population unless they left Ontario or died.

Medication use was recorded in patients $\geqslant 65$ years old if received any time after a sarcoidosis diagnosis for any duration of use. Primary care and subspecialty visits were recorded if patients had at least one visit with an applicable physician after the date of sarcoidosis diagnosis. In this way numerous visits to one physician or visits to more than one physician were captured.

\section{Analysis}

Analysis of disease trends was similar to that performed elsewhere [8]. The cumulative sarcoidosis prevalence was calculated from 1996 to 2015 by dividing the number of living patients meeting each sarcoidosis disease definition at the end of each fiscal year by the provincial population for that year. To allow for the identification of existing cases, we reviewed patient data back to 1991, but began reporting prevalence rates as of 1996.

Incident sarcoidosis rates were calculated between 1996 and 2014 by dividing new sarcoidosis patients by the provincial population at risk for sarcoidosis (total population minus those previously diagnosed). We began reporting incidence rates as of 1996, allowing a washout period of 5 years. We do not present incident rates after 2014 given there was not enough time to meet case definitions.

All-cause mortality rates were calculated for patients with sarcoidosis between 1996 and 2015 by dividing the total number of deaths per year among those with sarcoidosis by the total number with sarcoidosis in the corresponding fiscal year.

Prevalence and incidence rates were standardised for age and sex using the 2015 population in Ontario. Mortality rates were standardised for age and sex using the 2015 sarcoidosis population. Relative percentage changes for prevalence, incidence or mortality rates were calculated by dividing the rate of the most recent year by the rate of the earlier year. Percentage changes in disease rates were compared between 1996 and 2015 between age and sex groups using the Cochran-Mantel-Haenszel test. The Cochran-Armitage trend test was performed to assess for significance of trends over time. Rates between years were compared using the Chi-squared test and Fisher's exact test.

\section{Results}

In total, the numbers of patients between 1996 and 2015 meeting sarcoidosis, chronic sarcoidosis and sarcoidosis with histology definitions were 18550, 9199 and 3819, respectively. Descriptive statistics of the sarcoidosis, chronic sarcoidosis and sarcoidosis with histology cohorts are given in table 1. Most patients with sarcoidosis were aged 36-55 years at the time of diagnosis. Slightly more females were diagnosed with sarcoidosis than males. Prednisone, methotrexate and hydroxychloroquine were the most commonly prescribed treatments. Of specialists, pulmonologist were the most commonly visited. Approximately $11 \%$ of sarcoidosis patients were immigrants to Canada, with the most common countries of origin being India, Pakistan and Jamaica.

Comparisons between disease cohorts found patients with both chronic sarcoidosis and sarcoidosis with histology more commonly received prednisone, methotrexate and azathioprine than patients with sarcoidosis. Those included in the sarcoidosis with histology cohort were more commonly male, of high-income quintile and urban dwelling compared with the other sarcoidosis cohorts (table 1).

The frequency of sarcoidosis subgroups as documented by ICD-9/10 coding is shown in table 2 .

The age- and sex-standardised prevalence of sarcoidosis increased from 66 cases per 100000 in 1996 to 143 cases per 100000 in 2015 . This represents a $116 \%$ relative increase $(\mathrm{p}<0.0001)$. The prevalence increase appeared to be continual across the study period (figure 1a). Both males and females experienced an increase in age-standardised sarcoidosis prevalence between 1996 and 2015, but the increase was significantly higher in males (males $136 \%$ relative increase versus females $99 \%$ relative increase; $\mathrm{p}<0.0001$ ). The largest growth in sarcoidosis prevalence rates was in those aged $36-45$ years $(p=0.001)$ (figure $2 a)$.

The age- and sex-standardised prevalence of chronic sarcoidosis increased from 36 cases per 100000 in 1996 to 70 cases per 100000 in 2015 ( $p<0.0001$, for trend) (supplementary figure E1). The age- and sex-standardised prevalence of sarcoidosis with histology increased from 11 cases per 100000 in 1996 to 30 cases per 100000 in 2015 ( $\mathrm{p}<0.0001$, for trend) (supplementary figure E1). 
TABLE 1 Demographics and medication use in the prevalence cohorts of sarcoidosis, chronic sarcoidosis and sarcoidosis with histology from 1996 to 2015

\begin{tabular}{|c|c|c|c|}
\hline & Sarcoidosis & Chronic sarcoidosis & Sarcoidosis with histology \\
\hline Subjects & 18550 & 18550 & 3819 \\
\hline \multicolumn{4}{|l|}{ Age (median (IQR)) at diagnosis years } \\
\hline Female & $49(18)$ & $50(18)$ & $50(18)$ \\
\hline Male & $45(18)$ & $45(18)$ & $45(18)$ \\
\hline \multicolumn{4}{|l|}{ Age at diagnosis } \\
\hline $18-25$ years & $422(2.3)$ & $154(1.7)$ & $62(1.6)$ \\
\hline $26-35$ years & $3083(16.6)$ & $1473(16.0)$ & $592(15.5)$ \\
\hline $36-45$ years & 4855 (26.2) & $2481(27.0)$ & $990(25.9)$ \\
\hline $46-55$ years & $4713(25.4)$ & $2409(26.2)$ & $1039(27.2)$ \\
\hline $56-65$ years & $3268(17.6)$ & $1687(18.3)$ & $715(18.7)$ \\
\hline$\geqslant 66$ years & 2209 (11.9) & $995(10.8)$ & $421(11.0)$ \\
\hline Female & $9856(53.1)$ & $4960(53.9)$ & $1942(50.9)$ \\
\hline \multicolumn{4}{|l|}{ Income quintile } \\
\hline 1 (lowest) & 3567 (19.2) & $1736(18.8)$ & 737 (19.3) \\
\hline 2 & $3604(19.4)$ & $1804(19.6)$ & $727(19.0)$ \\
\hline 3 & $3720(20.1)$ & $1857(20.2)$ & 732 (19.2) \\
\hline 4 & $3702(20.0)$ & $1806(19.6)$ & 738 (19.3) \\
\hline 5 (highest) & $3814(20.1)$ & $1934(21.0)$ & $866(22.7)$ \\
\hline Rural (versus urban) & $2562(13.8)$ & $1185(12.9)$ & $446(11.7)$ \\
\hline \multicolumn{4}{|l|}{ Physician type ${ }^{\#}$} \\
\hline Family medicine & 18339 (98.9) & 9151 (99.5) & 3785 (99.1) \\
\hline Pulmonology & 15805 (85.2) & 8486 (92.2) & 3517 (92.1) \\
\hline Cardiology & 14716 (79.3) & 7753 (84.3) & $3230(84.6)$ \\
\hline Dermatology & $8053(43.4)$ & $4482(57.8)$ & $2103(55.1)$ \\
\hline Internal medicine & $13699(73.8)$ & $7216(78.4)$ & 2882 (75.5) \\
\hline Neurology & 6385 (34.4) & 3571 (38.8) & 1388 (36.3) \\
\hline Ophthalmology & 10904 (58.8) & $6144(66.8)$ & $2442(64.0)$ \\
\hline \multicolumn{4}{|l|}{ Medication } \\
\hline Azathioprine & $178(2.4)$ & $117(3.1)$ & $51(3.6)$ \\
\hline Biologicals & $19(0.2)$ & $9(0.2)$ & $<5(0.4)$ \\
\hline Hydroxychloroquine & 315 (4.3) & 210 (5.5) & $88(6.2)$ \\
\hline Leflunomide & $49(0.7)$ & $25(0.7)$ & $9(0.6)$ \\
\hline Methotrexate & $344(4.6)$ & $243(6.4)$ & $100(7.0)$ \\
\hline Mycophenolate & $66(0.9)$ & $44(1.2)$ & $25(1.8)$ \\
\hline Prednisone & 2989 (40.3) & $1800(47.1)$ & 640 (44.9) \\
\hline Immigrants & $1867(10.6)$ & $921(10.0)$ & 420 (11.0) \\
\hline \multicolumn{4}{|l|}{ Country of origin } \\
\hline India & 277 (1.5) & $131(1.4)$ & $55(1.4)$ \\
\hline Pakistan & $138(0.7)$ & $69(0.8)$ & $30(0.8)$ \\
\hline Jamaica & $130(0.7)$ & $83(0.9)$ & $37(1.0)$ \\
\hline Other ${ }^{+}$ & $1322(7.1)$ & $638(6.9)$ & $298(7.8)$ \\
\hline \multicolumn{4}{|l|}{ World region } \\
\hline South Asia & 586 (3.2) & 291 (3.2) & $121(3.2)$ \\
\hline Western Nations/Europe & $399(2.2)$ & $188(2.0)$ & $84(2.2)$ \\
\hline Caribbean & $258(1.4)$ & $149(1.6)$ & $26(0.7)$ \\
\hline Middle East/North Africa & $214(1.2)$ & $111(1.2)$ & $56(1.5)$ \\
\hline Sub-Saharan Africa & $168(0.9)$ & $84(0.9)$ & $39(1.0)$ \\
\hline Hispanic America & $138(0.7)$ & $67(0.7)$ & $26(0.7)$ \\
\hline East Asia and Pacific & $104(0.6)$ & $31(0.3)$ & $17(0.4)$ \\
\hline
\end{tabular}

Data are presented as $\mathrm{n}$ or $\mathrm{n}(\%)$, unless otherwise stated. IQR: interquartile range. \#: physician type is expressed as the total number of patients in the corresponding cohort seen by each physician group (patients could see more than one type of physician); ๆ: only included patients $\geqslant 65$ years of age $(7419,3823$ and 1423 total patients for sarcoidosis, chronic sarcoidosis and sarcoidosis with histology cohorts, respectively); ${ }^{+}$: the "other" category for country of origin reflects a combination of 119 different countries with at least one individual diagnosed with sarcoidosis in the described cohorts.

The age- and sex-standardised incidence of sarcoidosis decreased from 7.9 cases per 100000 in 1996 to 6.8 cases per 100000 in 2014, representing a 15\% relative decrease ( $\mathrm{p}=0.0009)$ (figure $1 \mathrm{~b})$. The trend of decreasing incidence was statistically significant $(\mathrm{p}<0.0001)$. A $30.3 \%$ relative decline in sarcoidosis incidence 


\begin{tabular}{|c|c|}
\hline \multicolumn{2}{|l|}{ 1996-2001 (ICD-9 codes) } \\
\hline Hospitalisation events & 6338 \\
\hline Sarcoidosis & 6318 (99.7) \\
\hline Sarcoidosis meningitis & $20(0.3)$ \\
\hline \multicolumn{2}{|l|}{$2002-2015$ (ICD-10 codes) } \\
\hline Hospitalisation events & 6681 \\
\hline Sarcoidosis of lung & $2368(35.4)$ \\
\hline Sarcoidosis of lymph nodes & $213(3.2)$ \\
\hline Sarcoidosis of lung and lymph nodes & $188(2.8)$ \\
\hline Sarcoidosis of skin & $82(1.2)$ \\
\hline Sarcoidosis of other and combined sites & $1015(15.2)$ \\
\hline Sarcoidosis, unspecified & $2896(43.3)$ \\
\hline Cranial nerve palsies in sarcoidosis & $51(0.8)$ \\
\hline Myositis in sarcoidosis & $7(0.1)$ \\
\hline
\end{tabular}

Data are presented as $\mathrm{n}$ or $\mathrm{n}(\%)$. A single patient could contribute to more than one hospitalisation and more than one ICD code could be applied to the same admission.

between 1996 and 2015 was seen in females $(\mathrm{p}<0.0001)$ compared with a 5.5\% relative increase in males $(\mathrm{p}=0.47)$. Across age groups, a significant relative decline in sarcoidosis incidence was seen in patients aged 26-35 and 36-45 years, estimated at $44.4 \%(\mathrm{p}<0.0001)$ and $29.5 \%(\mathrm{p}=0.001)$, respectively (figure $2 \mathrm{~b})$.

The age- and sex-standardised incidence of chronic sarcoidosis decreased from 4.0 cases per 100000 in 1996 to 3.1 cases per 100000 in 2013 ( $p<0.0001$, for trend). The age- and sex-standardised incidence of sarcoidosis with histology increased from 1.7 cases per 100000 in 1996 to 1.8 cases per 100000 in 2013 ( $\mathrm{p}=0.73$, for trend) (supplementary figure E1).

Age- and sex-standardised all-cause mortality in patients with sarcoidosis increased from $1.15 \%$ in 1996 to $1.47 \%$ in 2015 , representing a $28 \%$ increase $(p=0.02)$ (figure $1 c)$. The overall trend in mortality over the study period was not statistically significant $(\mathrm{p}=0.39)$. A $46 \%$ relative increase in mortality rate was seen in males with sarcoidosis between 1996 and 2015 ( $\mathrm{p}=0.01$ ), whereas in females the increase in mortality was $15 \%(\mathrm{p}=0.32)$. Patients diagnosed with sarcoidosis at age $46-55$ years $(\mathrm{p}=0.06)$ and $\geqslant 66$ years $(\mathrm{p}=0.08)$ had the largest increase in mortality between 1996 and 2015 (figure 2c).

In the chronic sarcoidosis cohort, age- and sex-standardised mortality increased from $0.95 \%$ to $1.42 \%$ ( $\mathrm{p}=0.0008$, for trend). In the sarcoidosis with histology cohort, age- and sex-standardised mortality decreased from $1.85 \%$ to $1.41 \%$ ( $\mathrm{p}=0.04$, for trend) (supplementary figure $\mathrm{E} 1$ ).

The epidemiology and mortality of the sarcoidosis, chronic sarcoidosis and sarcoidosis with histology cohorts at the beginning and end of the study time period is presented in figure 3 .

\section{Discussion}

Using a large, complete population, we estimated trends in prevalence, incidence and mortality of sarcoidosis from 1996 to 2015 in Ontario, Canada. Our study represents a uniquely comprehensive report on the epidemiology of sarcoidosis in Canada and characterises the epidemiology of sarcoidosis subpopulations (chronic sarcoidosis and sarcoidosis with histology). Sarcoidosis appears to impact patients of all ages, socioeconomic status and ethnicity. However, the burden of sarcoidosis appears to be changing; we found the prevalence of sarcoidosis to be rising fastest in males, with higher disease incidence rates among males in recent years and declining incidence rates in females. Although age- and sex-adjusted mortality rates in patients with sarcoidosis remained relatively stable during the study period, mortality increased significantly in patients with chronic sarcoidosis. These results serve to inform both clinicians and health policy makers of the current state and evolving trends of sarcoidosis disease epidemiology.

The Canadian healthcare system provides universal health coverage to all citizens, allowing for an inclusive and accurate representation of sarcoidosis epidemiology. Health administration studies performed in European countries with universal healthcare have shown similar sarcoidosis disease rates to those reported here. Applying similar case definitions, ARKEMA et al. [7] reported a sarcoidosis prevalence in Sweden of 160 cases per 100000 and incidence of 11.5 cases per 100000 using health services data. Although Sweden provides universal health coverage, only patients seen by specialists were captured, which may fail to identify patients with milder forms of disease managed by primary care physicians [7]. Our results comprise all types of physician visits (primary practitioners and specialists). 

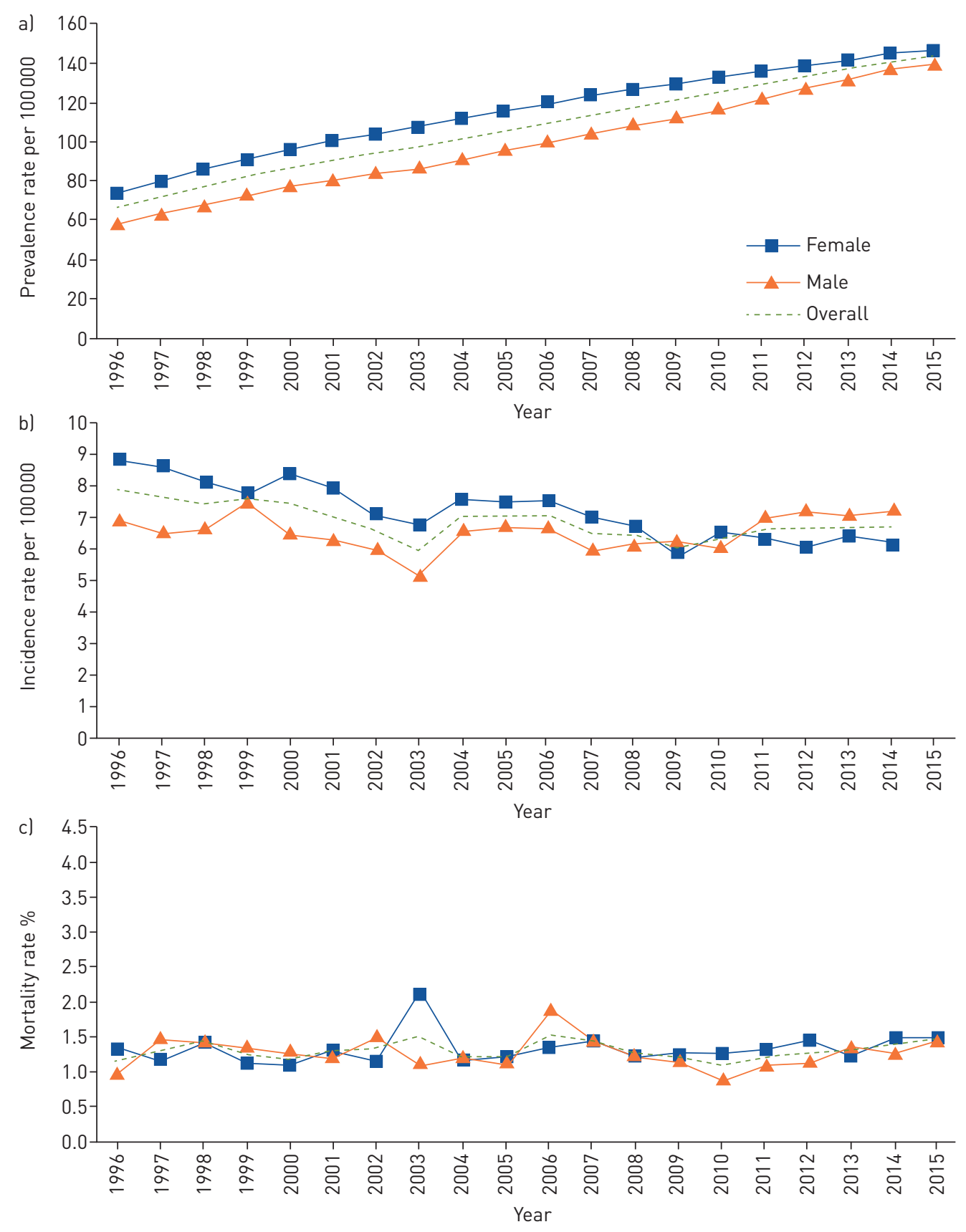

FIGURE 1 Age-standardised a) prevalence, b) incidence and c) mortality of patients with sarcoidosis in Ontario, Canada. Both prevalence and incidence rates are expressed in cases per 100000 . Mortality rate is expressed as percentage of patients susceptible.

In certain parts of USA, the prevalence of sarcoidosis has been reported to be on the rise [9]. BAUGHMAN et al. [4] reported the prevalence of sarcoidosis in the USA as around 60 cases per 100000 in 2013, substantially lower than that reported here. Although they interrogated a database of over 33 million individuals, only individuals with certain health insurance coverage were included in this study, which may underestimate sarcoidosis prevalence. Historically, racial minorities are less likely to have health insurance and have been reported to have higher rates of sarcoidosis $[4,5]$. We found the prevalence of sarcoidosis to be rising, despite a slight reduction in disease incidence over time. We suspect this is as a result of the low mortality rate compared with disease incidence and few patients leaving the province over time.

More females than males were diagnosed with sarcoidosis in our cohort, although the difference between the sexes was smaller than previous studies [10]. The gap in disease prevalence between males and females appears 

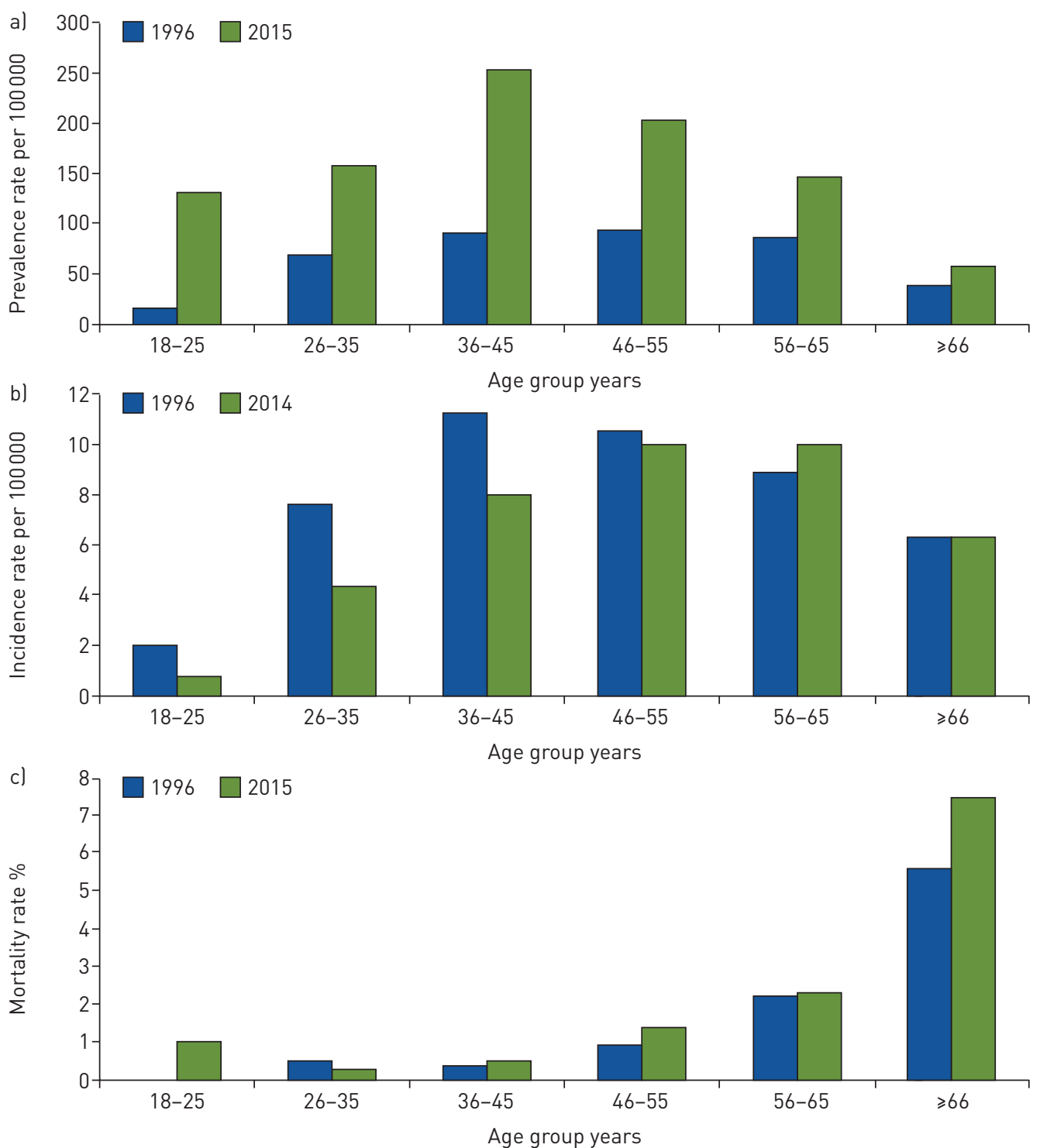

FIGURE 2 Sex-standardised a) prevalence, b) incidence and c) mortality of sarcoidosis by age group at the beginning and end of the study time period. Mortality is reported as the rate among individuals with sarcoidosis.

to be closing, with higher sarcoidosis incidence rates among males in recent years and decreasing incidence among females. Although the reasons for these trends are unclear, one potential explanation is an increase in occupational exposures in males compared with females. Exposure to inorganic dusts and building materials has been associated with sarcoidosis [11]. Ontario's construction industry has increased by 50\% since 2002 and employs nearly half a million Canadians, with $87 \%$ of employees being male [12]. Further research focused on exploring risk factors for sarcoidosis in Ontario might help to better understand this disease trend.

A lower percentage of sarcoidosis with histology patients were females compared with the other disease cohorts. It is unclear why fewer females underwent a biopsy as part of their diagnostic evaluation. Previous studies have found a higher proportion of males with end-stage pulmonary fibrosis related to sarcoidosis, which may support this hypothesis [13]. Alternatively, sex inequalities in the performance of diagnostic procedures may exist. Females have reported higher unmet healthcare needs in Canada compared with males, despite a universal healthcare system [14]. Additional research is needed to investigate whether these findings represent a true disparity in access to diagnostic testing or differences in disease presentation.

Sarcoidosis has been historically stated to develop predominantly in the second and third decade of life [1]. We found patients $>55$ years of age at the time of diagnosis comprised $<30 \%$ of sarcoidosis cases, with the majority of patients being in the third and fourth decade of life. BAUGHMAN et al. [4] reported that $>50 \%$ of patients in their recent cohort were $>55$ years of age at the time of diagnosis. We speculate the higher 


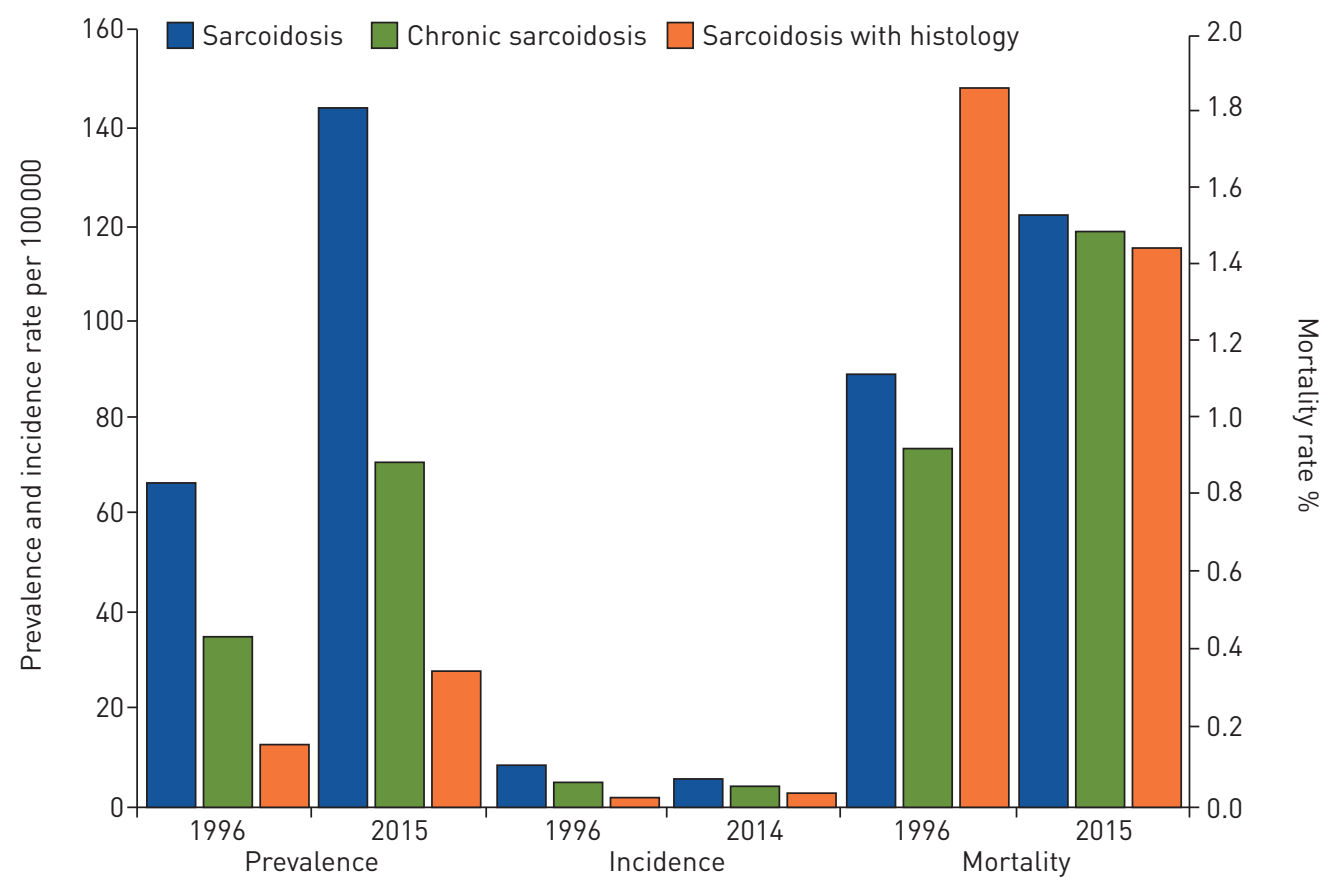

FIGURE 3 Epidemiology and mortality of the sarcoidosis, chronic sarcoidosis and sarcoidosis with histology cohorts at the beginning and end of the study time period.

proportion of sarcoidosis patients aged $>55$ years in that study may be due to a shorter look-back study time period ( 3 years); patients may have been diagnosed with sarcoidosis earlier in life, but if records were only examined within a short timeframe, prevalent cases may be inappropriately labelled as incident cases, falsely raising the age at diagnosis. We reviewed physician claims for 5 years prior to labelling incident cases and reviewed a cohort spanning nearly 20 years, which we suspect allowed for a more accurate estimate of incidence trends. That said, we found those aged 56-65 years to have the highest sarcoidosis incidence rates in recent years, with decreasing incidence rates in the younger age groups.

Of all the sarcoidosis patients we identified between 1996 and 2015, >10\% were immigrants to Canada. Between 2006 and 2011 the leading countries of origin of immigrants to Canada were the Philippines, China and India [15]. However, the countries of origin with the highest sarcoidosis prevalence were India, Pakistan and Jamaica. The high frequency of cases from Southeast Asia was unexpected, as Southeast Asians are not reported to be an ethnic group with strong associations with sarcoidosis [16]. Immigrants make up 20.6\% of the Canadian population and those living with sarcoidosis may have unique healthcare needs [17].

Previous reports evaluating the mortality of sarcoidosis in the USA have described increasing mortality rates over time, with higher mortality in females with sarcoidosis compared with males [18]. We found mortality rates in patients with sarcoidosis to be relatively stable over time, with similar annual mortality rates between males and females. However, patients within the chronic sarcoidosis cohort experienced a significant increase in mortality rates during the study period. Further research focused specifically on this higher risk population is needed to evaluate for risk factors associated with adverse health outcomes.

Several limitations of this research warrant mentioning. Despite the use of ICD-9/10 sarcoidosis-specific diagnostic codes, it is possible that patients were mislabelled as having sarcoidosis when they did not. Patients with mild forms of sarcoidosis who do not present to healthcare professionals for medical care would also be under-represented in this cohort. The presence of two or more sarcoidosis claims has been reported to capture the vast majority of sarcoidosis cases in a well-characterised cohort, although the specificity of this definition may be less optimal [7]. UNGPRASERT et al. [19] reported a positive predictive value of $76.5 \%$ for two or more sarcoidosis claims for confirmed cases in a small US cohort. To improve upon this, we developed cohorts with five or more sarcoidosis claims and biopsy-associated claims in an effort to enhance specificity. While our chronic sarcoidosis definition is believed to have face validity, we cannot be certain that a higher number of physician claims and longer duration of follow-up equates to disease chronicity or activity, due to the limitations of health administrative data.

We were limited to reviewing medication use in patients $\geqslant 65$ years of age. Although we report the burden of sarcoidosis among Canadian immigrants, we were limited to data regarding country of origin as 
opposed to self-reported ethnicity. Other important variables were not captured by the available databases, including pulmonary function testing, imaging and biopsy results.

Given the presented results were generated from a universal healthcare system, the reported patient outcomes may not be generalisable. Extrapolating the reported mortality trends to countries where healthcare access is limited may be flawed, as mortality rates may be higher in individuals without health insurance. However, we believe these results have external validity given the multiethnic cohort, which is similar to other North American populations.

Strengths of this research include the large, complete and heterogeneous population. We evaluated the epidemiology and mortality of three sarcoidosis cohorts, incorporating the performance of a tissue biopsy, which is often lacking in other health services research. We believe that the distribution of patients across disease cohorts is consistent with what is seen clinically, providing some degree of internal validity.

In conclusion, we report a large, population-based study of prevalence, incidence and mortality trends of sarcoidosis in Canada. The prevalence of sarcoidosis is rising, with an apparent shifting trend in disease burden from females to males. Mortality in patients suffering from chronic sarcoidosis is increasing. Additional research regarding risk factors for disease development and adverse health outcomes within the sarcoidosis population in Canada is needed to inform patients, healthcare providers and health policy experts.

Author contributions: L.M. Fidler, M. Balter, J.H. Fisher, M.B. Stanbrook and A. Gershon contributed to the conception and design of the performed research. L.M. Fidler, T. To and A. Gershon were involved in data analysis. All authors were responsible for data interpretation, drafting of the manuscript and approval of the final version.

Conflict of interest: None declared.

Support statement: This research was supported financially by a Mount Sinai Hospital research fund derived from patient donations. Funding information for this article has been deposited with the Crossref Funder Registry.

\section{References}

$1 \quad$ Iannuzzi MC, Rybicki BA, Teirstein AS. Sarcoidosis. N Engl J Med 2007; 357: 2153-2165.

2 Deubelbeiss A, Gemperli C, Schindler C, et al. Prevalence of sarcoidosis in Switzerland is associated with environmental factors. Eur Respir J 2010; 35: 1088-1097.

3 Arkema EV, Cozier YC. Epidemiology of sarcoidosis: current findings and future directions. Ther Adv Chronic Dis 2018; 9: 227-240.

4 Baughman RP, Field S, Costabel U, et al. Sarcoidosis in America. Analysis based on health care use. Ann Am Thorac Soc 2016; 13: 1244-1252.

5 Kirby JB, Kaneda T. Unhealthy and uninsured: exploring racial differences in health and health insurance coverage using a life table approach. Demography 2010; 47: 1035-1051.

6 Statistics Canada. Canada's Population Estimates, Third Quarter 2018. 2018. www150.statcan.gc.ca/n1/ daily-quotidien/181220/dq181220c-eng.htm Date last accessed: February 23, 2019.

7 Arkema EV, Grunewald J, Kullberg S, et al. Sarcoidosis incidence and prevalence: a nationwide register-based assessment in Sweden. Eur Respir J 2016; 48: 1690-1699.

8 Gershon AS, Wang C, Wilton AS, et al. Trends in chronic obstructive pulmonary disease prevalence, incidence, and mortality in Ontario, Canada, 1996 to 2007. A population-based study. Arch Intern Med 2010; 170: 560-565.

9 Erdal BS, Clymer BD, Yildiz VO, et al. Unexpectedly high prevalence of sarcoidosis in a representative U.S. metropolitan population. Respir Med 2012; 106: 893-899.

10 Baughman RP, Teirstein AS, Judson MA, et al. Clinical characteristics of patients in a case control study of sarcoidosis. Am J Respir Crit Care Med 2001; 164: 1885-1889.

11 Barnard J, Rose C, Newman L, et al. Job and industry classifications associated with sarcoidosis in A Case-Control Etiologic Study of Sarcoidosis (ACCESS). J Occup Environ Med 2005; 47: 226-234.

12 BuildForce Ontario. Construction and Maintenance Looking Forward, 2018-2027. 2018. www. constructionforecasts.ca/sites/forecast/files/highlights/2018/2018_ON_Constr_Maint_Looking_Forward.pdf Date last accessed: February 23, 2019.

13 Nardi A, Brillet PY, Letoumelin P, et al. Stage IV sarcoidosis: comparison of survival with the general population and causes of death. Eur Respir J 2011; 38: 1368-1373.

14 Socias MA, Koehoorn M, Shoveller J. Gender inequalities in access to health care among adults living in British Columbia, Canada. Womens Health Issues 2016; 26: 74-79.

15 Statistics Canada. Immigration and Ethnocultural Diversity. 2017. https://www12.statcan.gc.ca/census-recensement/ 2016/rt-td/imm-eng.cfm Date last accessed: August 7, 2019.

16 Young RC Jr, Hackney RL Jr, Harden KA. Epidemiology of sarcoidosis: ethnic and geographic considerations. J Natl Med Assoc 1974; 66: 386-388.

17 Ng E, Wilkins R, Gendron F, et al. Dynamics of Immigrants' Health in Canada: Evidence from the National Population Health Survey. 2005. https://www150.statcan.gc.ca/n1/pub/82-618-m/82-618-m2005002-eng.htm Date last accessed: February 23, 2019.

18 Swigris JJ, Olson AL, Huie TJ, et al. Sarcoidosis-related mortality in the United States from 1988 to 2007. Am J Respir Crit Care Med 2011; 183: 1524-1530.

19 Ungprasert P, Matteson EL, Crowson CS. Accuracy of diagnostic coding for sarcoidosis in electronic databases: a population-based study. Lung 2017; 195: 713-715. 